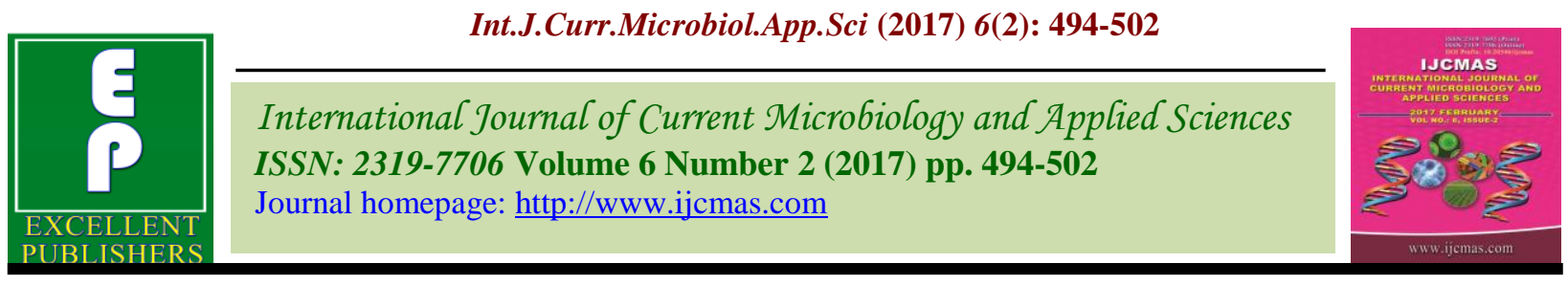

Original Research Article

http://dx.doi.org/10.20546/ijcmas.2017.602.056

\title{
Environmental Influence under Off-season Production on Yield and Quality Attributes of Mango
}

\author{
A. Kaviarasu ${ }^{1}$, S. Balakrishnan ${ }^{2}$ and K. Indirakumar ${ }^{3}$ \\ ${ }^{1}$ Department of Horticulture, Thanthai Roever Institute of Agriculture and \\ Rural Development, Perambalur- 621 115, India \\ ${ }^{2}$ Department of fruit science, Horticulture College and Research Institute, \\ Tamil Nadu - 641003, India \\ ${ }^{3}$ Department of Agricultural Entomology, Thanthai Roever Institute of Agriculture and Rural \\ Development, Perambalur - 621 115, India \\ *Corresponding author
}

\section{A B S T R A C T}

\section{Keywords}

Off-season, yield attributes, Quality attributes and Meteorological parameter.

\section{Article Info}

Accepted: 15 January 2017 Available Online: 12 February 2017
An experiment was conducted at the State Horticulture Farm Kanyakumari in eighteen regular off-season bearing varieties where flowering is observed during August, September, October, November months with the fruiting period during December-January were selected for this study. The off-season bearing varieties are Neelum, Kalepad, Bangalora, Banganapalli, Himayuddin, Nadan, Panchavarnam, Rumani, Dilpasand, Kalkachi, Jehangir, Pairi, Panikanadan, Khudadad, Athimathuram, Mulgoa, Alphonso and Surangudi. The off-season flowering is a peculiar phenomenon in Tamilnadu because continuous flowering, fruiting and vegetative production can be seen simultaneously in Kanyakumari. The off-season flowering is usually observed during July-August and harvesting of fruits is usually done during December-January. While most of the varieties shows some peculiar characters in fruit yield and quality attributes it plays a vital role in post harvest self- life in preserving quality. The highest yield was recorded in Bangalora $(520.80 \mathrm{Kg} / \mathrm{tree})$. It recorded maximum fruit length $(22.60 \mathrm{~cm})$, fruit girth $(33.30 \mathrm{~cm})$, fruit volume $(469.50 \mathrm{ml})$, fruit weight $(471.50 \mathrm{~g})$ and number of fruits per tree $(560.00)$. Bangalora recorded the highest TSS $\left(20.50^{\circ}\right.$ Brix), total sugars $(18.16 \%)$, reducing sugars $(7.83 \%)$, non reducing sugars $(10.34 \%)$, carotene content $(60.25 \mathrm{mg} / \mathrm{kg})$, titrable acidity $(0.49 \%)$ and ascorbic acid content $(37.18 \mathrm{mg} / 100 \mathrm{~g})$. The maximum temperature (29.9 to $\left.32.9^{\circ} \mathrm{C}\right)$, diurnal variation $\left(6.9^{\circ} \mathrm{C}\right)$, relative humidity $(76.6 \%)$, rainfall $(159.53 \mathrm{~mm})$, wind velocity $(6.13 \mathrm{kmph})$ and sunshine hours $(437.05)$ were highly conducive for off-season flowering in Kanyakumari.

\section{Introduction}

The mango (Mangifera indica L. Anacardiacea) is one of the choicest fruit crops of tropical and sub-tropical regions of the world, especially in Asia. Its popularity and importance can easily be realized by the fact that it is often referred as 'King of fruits' in the tropical world (Singh, 1996). The production of off-season cropping in mango which coincides with November-December months should be properly considered for 
quality aspects also as fruit development during rainy/cool season months will be of generally inferior in quality because of prevalence of lower quantum of heat units (Ananthanarayan and Anchanam Alagia Pillai, 1968). Whereas flowering is the first of several events that set the stage for mango production each year off-season cropping of certain tracts of Tamil Nadu.

Besides the normal crop that matures from May to August, the trees of certain varieties also mature an off-season crop from October to January. Neelum, Bangalora, Calcutta or Bengal Barasmasi, Kintalvanipeta, and Ali Pasand have been mentioned as varieties bearing two or more crops in succession during certain years and extend their bearing period almost till December and sometimes throughout the year. This has been attributed to the peculiar seasonal conditions and the fairly well distributed rainfall of the area (Chacko and Randhawa, 1971) (Sundararaj et al., 1972). In a study to find out the cause of differential flowering in mango in the island ecosystem by Damodaran et al., (2006) reported that the specific bands obtained were responsible for genes associated with differential flowering behaviour and is due to introgression of genes during hybridization. At Coimbatore, the varieties Peter and Baneshan have been reported as flowering in the off-season. At Kodur, the variety Ambalavi produced five blossom crops between September and the following may except Neelum, Kintalvanipeta, Manoranjan and Willard produced three crops of blossoms. The clonal progeny of an offseason bearing tree at Tenali was found to behave tree at Tenali was found to behave like the parent tree. These facts suggested that the off-season bearing, though a varietal character, was induced by favourable seasonal and climatic conditions. Climate change has emerged as a major challenge influencing agricultural production in the country. Mango crops, being perennial in nature, have to face the impact of climate change even during a single generation or in a standing crop. Hence it is important that the impact of climate change is understood well. Mangoes are grown in ecologically sensitive areas such as coastal belts, hilly areas and areas with high rainfall and high humidity. This crop is of high economic value contributing substantially to the agricultural exports at global and national levels. These are grown in large areas in Kerala, Karnataka, Tamil Nadu, Andhra Pradesh, Maharashtra, West Bengal and Assam. The mango crops provide sustenance to the millions of people. Weather variables such rainfall, evapotranspiration, temperature, solar radiation, sunshine hours, relative humidity and wind velocity influence the yield potential of mango. The present study was undertaken for the seasonal effect of climatic condition under off-season production in yield and quality attributes of mango.

\section{Materials and Methods}

The present experiment was conducted at the State Horticulture Farm Kanyakumari, Tamil Nadu Investigation on off-season flowering, fruiting behaviour, effect of growth, physiological changes, yield and yield attributes of eighteen mango varieties was under taken during the period between August 2010 and January 2011. The off-season bearing varieties are Neelum, Kalepad, Bangalora, Banganapalli, Himayuddin, Nadan, Panchavarnam, Rumani, Dilpasand, Kalkachi, Jehangir, Pairi, Panikanadan, Khudadad, Athimathuram, Mulgoa, Alphonso and Surangudi. Experiments were arranged in randomized block design replicated three times. Five trees were used in each variety for replication 25 years old trees were chosen for the study. From each tree, 20-30 samples were taken at monthly intervals and quality analysis was carried out to assess the fruit 
quality by adopting the standard procedures five fruits from each treatment were harvested at uniform maturity and allowed to ripe naturally in the room temperature. At edible ripening stage, the fruits were cut and the pulp were mixed together for performing the quality analysis. Observations on yield attributes, quality attributes and weather parameters were recorded.

\section{Assessment of fruit yield characters on off- season mango}

Off-season fruits were assessed for determining the following yield parameters. Fruit length and grith were determined by the means of expressed in centimeter. Fruit volume was determined by the means of expressed in milliliter. The number of fruits harvested per tree was counted and the total was expressed as number of fruits per tree. Fruit weight, Peel weight, Pulp Weight and Stone Weight were randomly selected from each tree and the average weight of the pulp was calculated and expressed in gram. The ratio of Stone to pulp was calculated based on the analysis of total stone and total pulp. Total weight of the fruits harvested per tree was recorded and expressed in kilograms (Kg/tree). Specific gravity of fruit was calculated as (Density of mango fruit/ Density of water), Density was calculated as (mass/volume). The number of days to ripen after harvest of fruits was recorded and expressed in number.

Estimation of important quality attributes on off-season mango

Fully matured representative figures were allowed for natural and uniform ripening in the room temperature. These fruits were assessed for determined the following quality parameters. The total soluble solids were determined by using Carl-Zeiss hand refractrometer and expressed as degree Brix.
The total sugars, reducing and non-reducing sugars were estimated as per the methods suggested by Somogyi (1952) and expressed as percentage. The carotenoid content of the fruit was estimated by adopting the A.O.A.C. (1975) method and expressed as Milligrams/ Kilogram. Titrable acidity of the fruit was estimated by adopting the method of A.O.A.C. (1975) by titrating against N/10 $\mathrm{KOH}$ using phenolphthalein indicator and expressed in terms of percentage of citric acid. Ascorbic acid content was estimated by A.O.A.C. (1975) by using 2, 6dichlorophenol indophenols dye and the value was expressed as milligrams/100gram.

\section{Meteorological Parameters from flowering to maturity}

Monthly maximum temperature ${ }^{\circ} \mathrm{C}$ and Monthly minimum temperature ${ }^{\circ} \mathrm{C}$ were expressed in terms of (Celsius). Relative humidity was expressed in terms of percentage. Rainfall was expressed in terms of mille meter. Wind velocity was expressed in kilo meters per hour. Sunshine hours were short day and long day hours. Day and night temperature was calculated as Diurnal variation (Maximum temperature - Minimum temperature).

\section{Results and Discussion}

\section{Effect of seasonal difference on off-season} fruiting yield parameters of mango

The maximum fruit length $(22.60 \mathrm{~cm})$, fruit girth $(33.30 \mathrm{~cm})$, fruit volume $(469.50 \mathrm{ml})$, fruit weight $(471.50 \mathrm{~g})$ and pulp weight $(365.00 \mathrm{~g})$ were recorded in Bangalora. Nadan recorded maximum peel weight (79.50 g) and Athimathuram recorded the maximum stone: pulp ratio of 10.84 (Table 1). Due to seasonal and varietal variation of fruit characters in post-harvest study were recorded in Bangalora, Neelum, Himayuddin followed 
by Kalepad were influenced by different seasonal effects on climatic changes and biological wonders in Kanyakumari reported by (Kennedy et al., 2009a) (Kennedy et al., 2009b) and (Parthiban et al., 2009). Maximum yield performance of Bangalora was due to their prolific nature and adaptability to Kanyakumari regions of Tamil Nadu. Similar variations in yield in the offseason bearing nature of mango varieties were recorded at Erode (Sundaram, 2007) and at Sangareddy (Lakshminarayanareddy et al., 2009). Bangalora recorded the maximum number of fruits per tree (560.00) and fruit yield per tree (520.80). Similar variation in fruit yield per tree had also been reported by Kumar and Kavino (2009).

\section{Effect of seasonal difference on off-season fruiting quality parameters of mango}

The maximum total soluble solids $\left(20.50^{\circ}\right.$ brix), total sugars $(18.16 \%)$, reducing sugars (7.83\%), non reducing sugars (10.34\%), carotene $(60.25 \mathrm{mg} / \mathrm{kg})$ and ascorbic acid content $(37.18 \mathrm{mg} / 100 \mathrm{~g})$ were recorded in Bangalora. The available photosynthates required for fruit development are diverted to improve the TSS. Similar finding was reported by Hassan et al., 2004; Khattab et al., 2006 and Shaban, 2009. The maximum titrable acidity content was recorded in Surangudi (0.49\%) (Table 2). Srinivasan and Shanmugavelu (1971) reported that Low acidity of the fruits is one of the desirable qualities in mango. In mango, the quality is mainly judged by total soluble solids (TSS), total sugars, titrable acidity and total carotenoid content in fruits.

\section{Effect on off-season environmental changes in meteorological parameters}

The location of the experiment site might play a critical role in induction of off- season flowering as experiences have shown that off- season flowering cannot be seen as a uniform phenomenon in all mango growing regions. In the present case, it is surrounded by hills on three sides presenting a different microclimate, favouring off-season cropping than the other neighboring parts. The fluctuation between the maximum and minimum relative humidity was found to be wide during the main season, while it was narrow during the off-season. Ananthanarayanan and Pillai (1968) while studying the off-season bearers at Kanyakumari also opined that high humidity and rainfall prevailed during the off-season than the main season.

Monthly maximum temperature during August to January 2010-2011 ranged from $\left(29.9^{\circ} \mathrm{C}\right.$ to $\left.32.9^{\circ} \mathrm{C}\right)$. While, the monthly minimum temperature ranged from $\left(23.5^{\circ} \mathrm{C}\right.$ to $25.2^{\circ} \mathrm{C}$ ) in the experimental location. These temperature regimes might be probably conducive for flowering and fruit setting during the off-season. Another important factor is the absence of very heavy rain during these months as rains, fogs or cloudy weather at the time of flowering prevents the setting of fruits and favour the development of pests and diseases similar finding was reported by Gangolly et al., 1957 and Gandhi, 1959. Relative humidity ranged between (71.6 to $80.6 \%$ ). There are many schools of thoughts ascribed to this phenomenon and it has observed by the mango research that a high relative humidity of $90 \%$; low night temperature during south west monsoon; a well distributed monsoon rains may be responsible for residual food reserve conversion into flower primordial even in young shoots also (Richard Kennedy et al., 2009a). The per cent soil moisture was also high during off-season because of high rainfall. The location receives nearly (400.9 $\mathrm{mm}$ ) of rain fall during the off-season flowering period and which is followed by low rainfall (29.5) during subsequent months 
that favours better fruit setting Naik (1949) reported that dry and relatively rainless summer evidently helped the shoot to get the desired rest period for successful initiation of flower buds. Cloudy weather and rains during blossoming period reduced the crop indirectly by creating favourable conditions for pest and disease (Singh, 1957). Fairly well distributed rainfall may be the desirable feature for the production of a regular off-season crop in Kanyakumari (Lysander and Pillai 1957; Velappan and Shankar, 2001).

Table.1 Effect of seasonal difference on off-season fruiting yield parameters of mango

\begin{tabular}{|c|c|c|c|c|c|c|c|c|c|c|c|c|c|c|c|c|c|c|c|c|}
\hline 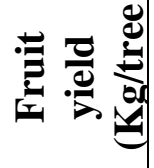 & ñ & ñ & $\begin{array}{l}\infty \\
\infty \\
\text { N } \\
\text { ñ }\end{array}$ & ז̊ & ڤ̊ & $\begin{array}{l}\mathfrak{n} \\
\hat{0} \\
\hat{0}\end{array}$ & $\begin{array}{l}\vec{y} \\
\infty \\
\stackrel{0}{2}\end{array}$ & $\begin{array}{l} \pm \\
\vdots \\
\infty\end{array}$ & 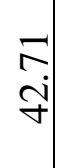 & $\frac{\tilde{s}}{n}$ & \begin{tabular}{l}
\multirow{2}{*}{} \\
$\stackrel{7}{+}$
\end{tabular} & $\begin{array}{l}n \\
0 \\
\grave{0}\end{array}$ & ح. & $\ddot{n}$ & ? & 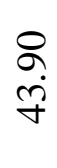 & $\stackrel{\infty}{\infty}$ & 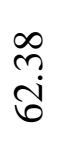 & 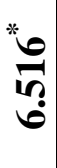 & 离 \\
\hline 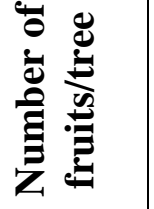 & $\begin{array}{l}8 \\
\dot{9} \\
\dot{+}\end{array}$ & 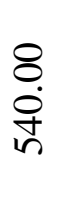 & $\begin{array}{l}8 \\
8 \\
0 \\
0 \\
\end{array}$ & 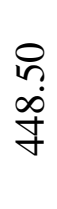 & 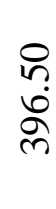 & ָ̊. & $\frac{n}{n}$ & ڤ̊n. & $\begin{array}{l}0 \\
0 \\
0 \\
\infty\end{array}$ & 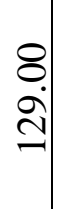 & 号 & 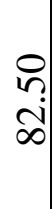 & $\begin{array}{l}8 \\
8 \\
\dot{0} \\
\end{array}$ & ñ? & กี่ & ஸ் & $\frac{n}{6}$ & $\begin{array}{l}\stackrel{8}{0} \\
\stackrel{0}{7}\end{array}$ & ma & बे \\
\hline 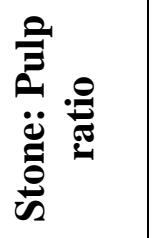 & $\begin{array}{l}\text { oे } \\
\dot{+}\end{array}$ & $\begin{array}{l}\text { bे } \\
\dot{r}\end{array}$ & రీ & $\stackrel{m}{m}$ & in & $\begin{array}{l}n \\
\stackrel{n}{7} \\
\text { in }\end{array}$ & ণิ & $\begin{array}{l}8 \\
\text { m. }\end{array}$ & 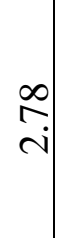 & $\begin{array}{l}\dot{J} \\
\dot{t}\end{array}$ & $\begin{array}{l}\hat{\sigma} \\
\dot{m}\end{array}$ & $\begin{array}{l}0 \\
\infty \\
i\end{array}$ & $\begin{array}{l}\hat{b} \\
\dot{n}\end{array}$ & $\vec{\nabla}$ & $\begin{array}{l}+ \\
0 \\
0\end{array}$ & $\begin{array}{l}\text { Wु } \\
\text { i }\end{array}$ & $\vec{z}$ & 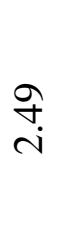 & $\begin{array}{l}\hat{\infty} \\
\tilde{\sigma} \\
0\end{array}$ & க) \\
\hline 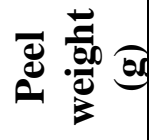 & $\stackrel{\circ}{\sim}$ & $\begin{array}{l}8 \\
\text { \& } \\
\dot{+}\end{array}$ & $\begin{array}{l}8 \\
8 \\
\dot{0}\end{array}$ & ֻ̊ & $\frac{8}{8}$ & 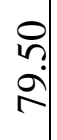 & $\begin{array}{l}8 \\
\dot{m}\end{array}$ & 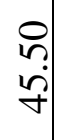 & 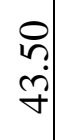 & $\begin{array}{l}8 \\
\varnothing \\
\dot{\sigma}\end{array}$ & $\begin{array}{l}8 \\
\dot{+}\end{array}$ & 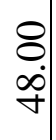 & $\begin{array}{l}8 \\
\text { n. } \\
\text { m. }\end{array}$ & $\begin{array}{l}8 \\
0 \\
0 \\
0\end{array}$ & $\begin{array}{l}8 \\
\text { ப் }\end{array}$ & 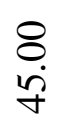 & $\frac{8}{3}$ & $\frac{\circ}{n}$ & m. & $\overline{8}$ \\
\hline 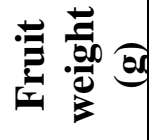 & $\begin{array}{l}8 \\
\text { ì } \\
\text { त }\end{array}$ & $\begin{array}{l}0 \\
n \\
\infty \\
n \\
n\end{array}$ & $\frac{?}{*}$ & 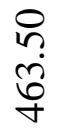 & $\begin{array}{l}8 \\
\qquad \\
\grave{n}\end{array}$ & $\begin{array}{l}0 \\
n \\
n \\
\infty \\
m\end{array}$ & 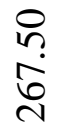 & 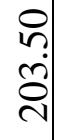 & $\begin{array}{l}8 \\
\text { ปे. } \\
\text { ปे }\end{array}$ & 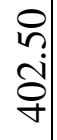 & 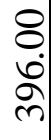 & $\begin{array}{l}8 \\
\dot{0} \\
\stackrel{7}{+}\end{array}$ & $\begin{array}{l}\stackrel{0}{2} \\
\stackrel{\sim}{\sim} \\
\stackrel{v}{ }\end{array}$ & $\begin{array}{l}0 \\
\text { ñ } \\
\frac{6}{n}\end{array}$ & $\begin{array}{l}8 \\
8 \\
0 \\
\text { †े }\end{array}$ & \begin{tabular}{l}
8 \\
\multirow{+}{\pm}{} \\
ป
\end{tabular} & $\begin{array}{l}8 \\
0 \\
1\end{array}$ & $\begin{array}{l}8 \\
\text { in } \\
\text { n }\end{array}$ & 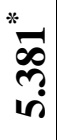 & ?n \\
\hline 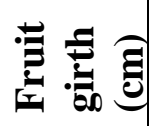 & \begin{tabular}{l}
$n$ \\
\multirow{2}{1}{}
\end{tabular} & $\begin{array}{l}\stackrel{0}{0} \\
\stackrel{d}{d}\end{array}$ & $\begin{array}{l}0 \\
m \\
m\end{array}$ & m & $\stackrel{n}{\stackrel{n}{2}}$ & $\begin{array}{l}n \\
0 \\
0 \\
\text { n. }\end{array}$ & 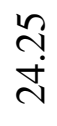 & $\stackrel{n}{\tilde{+}}$ & $\begin{array}{l}\text { 을 } \\
\text { הิ }\end{array}$ & กุ & 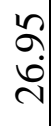 & 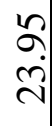 & $\stackrel{0}{\stackrel{0}{0}}$ & $\begin{array}{l}\text { กิ } \\
\text { ปู }\end{array}$ & $\stackrel{n}{n}$ & 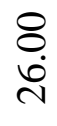 & $\begin{array}{l}8 \\
\text { ปี }\end{array}$ & $\frac{n}{\stackrel{n}{d}}$ & $\stackrel{*}{\text { f }}$ & $\stackrel{\varrho}{0}$ \\
\hline 灵总 & 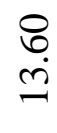 & $\begin{array}{l}\text { D } \\
\text { İ }\end{array}$ & $\begin{array}{l}8 \\
\text { i } \\
\text { in }\end{array}$ & $\begin{array}{l}0 \\
\stackrel{0}{n}\end{array}$ & $\begin{array}{l}\stackrel{0}{J} \\
\dot{ \pm}\end{array}$ & ñ & $\stackrel{n}{\sim}$ & $\begin{array}{l}n \\
\end{array}$ & 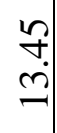 & $\begin{array}{l}\text { స̦ } \\
\text { ஸి }\end{array}$ & 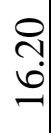 & $\begin{array}{l}n \\
0 \\
i\end{array}$ & 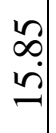 & $\begin{array}{l}n \\
\stackrel{n}{n}\end{array}$ & $\begin{array}{l}n \\
n \\
n\end{array}$ & $\begin{array}{l}\infty \\
\dot{ \pm} \\
\dot{1}\end{array}$ & $\stackrel{\mathfrak{a}}{\beth}$ & $\frac{n}{\mathfrak{d}}$ & مَn & $\bar{\Upsilon}$ \\
\hline 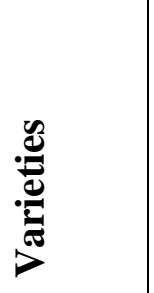 & $\frac{\Xi}{\mathbb{Z}}$ & $\frac{\text { है }}{\frac{0}{0}}$ & 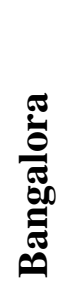 & 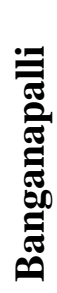 & 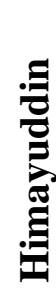 & $\begin{array}{l}\tilde{\tilde{T}} \\
\mathbf{Z} \\
\mathbf{Z}\end{array}$ & 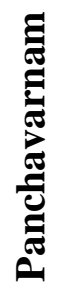 & 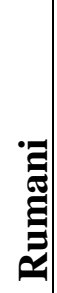 & 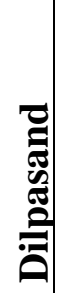 & 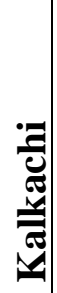 & 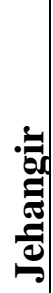 & : & 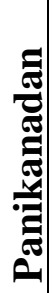 & 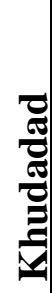 & 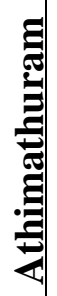 & 造 & $\frac{0}{\frac{2}{2}}$ & 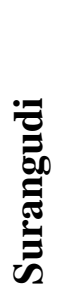 & 牙 & 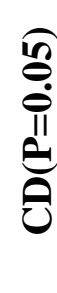 \\
\hline$\dot{\omega} \ddot{z}$ & - & $\sim$ & $\pi$ & $\checkmark$ & $n$ & 0 & $r$ & $\infty$ & $a$ & 0 & -1 & $\simeq$ & $\stackrel{m}{=}$ & $\Xi$ & $n$ & 4 & - & $\stackrel{\infty}{-}$ & & \\
\hline
\end{tabular}


Int.J.Curr.Microbiol.App.Sci (2017) 6(2): 494-502

Table.2 Effect of seasonal difference on off-season fruiting quality parameters of mango

\begin{tabular}{|c|c|c|c|c|c|c|c|c|c|c|c|c|c|c|c|c|c|c|c|c|}
\hline 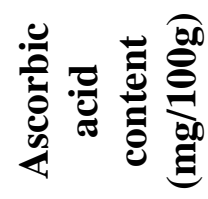 & $\begin{array}{l}\hat{\sigma} \\
\stackrel{m}{m}\end{array}$ & $\overline{6}$ & $\stackrel{\infty}{\stackrel{\infty}{n}}$ & $\begin{array}{l}\text { I. } \\
\dot{m}\end{array}$ & $\frac{\stackrel{\overbrace{}}{n}}{\dot{m}}$ & ลे. & $\stackrel{5}{m}$ & 宓 & 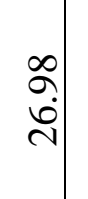 & तิ & $\begin{array}{l}\infty \\
\stackrel{\infty}{+} \\
\stackrel{2}{2}\end{array}$ & $\frac{\text { ก̦ }}{\dot{m}}$ & సి. & $\begin{array}{l}\infty \\
\text { ఫे } \\
\text { ते }\end{array}$ & $\begin{array}{l}\text { ते| } \\
\text { ते }\end{array}$ & సి & ते. & $\begin{array}{l}8 \\
\stackrel{8}{0} \\
\text { in }\end{array}$ & $\stackrel{\tilde{\pi}}{3}$ & \\
\hline 总 & ले & के & $\tilde{0}$ & $\stackrel{+}{+}$ & $\stackrel{q}{0} \cdot$ & ơ & $\stackrel{n}{\circ}$ & 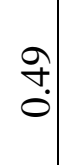 & $\stackrel{\infty}{+}$ & $\stackrel{?}{+}$ & $\begin{array}{l}0 \\
0 \\
0\end{array}$ & $\stackrel{m}{\circ}$ & $\stackrel{n}{\circ}$ & $\stackrel{\infty}{+}$ & 守 & $\stackrel{n}{\circ} \cdot$ & $\stackrel{f}{0}$ & $\stackrel{a}{0}$ & $\stackrel{\tilde{\Xi}}{0}$ & \\
\hline 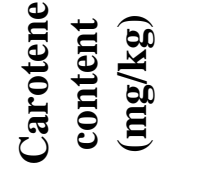 & $\stackrel{n}{\stackrel{n}{\circ}}$ & $\begin{array}{l}3 \\
\dot{b} \\
i\end{array}$ & 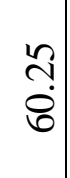 & $\begin{array}{l}\tilde{n} \\
\stackrel{n}{n} \\
n\end{array}$ & $\stackrel{n}{\stackrel{n}{+}}$ & $\stackrel{\oplus}{\mathrm{n}}$ & $\begin{array}{l}\stackrel{n}{0} \\
\dot{f}\end{array}$ & $\begin{array}{c}\infty \\
\stackrel{+}{q} \\
\dot{q}\end{array}$ & 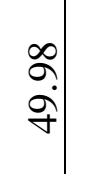 & $\begin{array}{l}n \\
\stackrel{n}{n}\end{array}$ & $\begin{array}{l}\infty \\
0 \\
i \\
i\end{array}$ & $\begin{array}{l}\stackrel{+}{+} \\
\stackrel{+}{n}\end{array}$ & 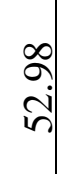 & $\begin{array}{l}\text { กิ } \\
\text { ஸे }\end{array}$ & $\begin{array}{l}0 \\
\stackrel{\vec{n}}{n}\end{array}$ & $\begin{array}{l}\hat{\hat{n}} \\
\hat{n}\end{array}$ & $\frac{\hat{0}}{\dot{n}}$ & $\begin{array}{l}\stackrel{o}{+} \\
\dot{q} \\
\dot{q}\end{array}$ & :ै & \\
\hline 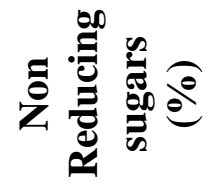 & $\vec{\sigma}$ & ?ֶ. & $\begin{array}{l}\stackrel{+}{?} \\
\stackrel{0}{0}\end{array}$ & 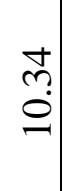 & $\begin{array}{l}\mathbb{b} \\
\infty \\
\infty\end{array}$ & ণ্ণి & $\begin{array}{l}\hat{b} \\
\infty \\
\infty\end{array}$ & $\begin{array}{l}\exists \\
\overrightarrow{+}\end{array}$ & $\begin{array}{l}\stackrel{\infty}{+} \\
+ \\
+\end{array}$ & $\begin{array}{l}3 \\
\infty \\
\infty\end{array}$ & $\begin{array}{l}\infty \\
0 \\
0\end{array}$ & $\begin{array}{l}\mathfrak{\sigma} \\
\infty \\
\infty\end{array}$ & $\stackrel{0}{n}$ & $\stackrel{m}{+}$ & $\vec{m}$ & $\stackrel{ }{ }$ & ?ొ: & ڤి & $\stackrel{\text { : }}{8}$ & \\
\hline 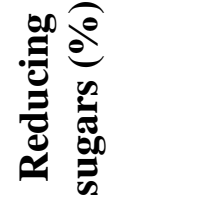 & $\stackrel{?}{\stackrel{P}{+}}$ & $\begin{array}{l}n \\
\stackrel{n}{p} \\
r\end{array}$ & 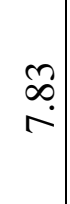 & $\begin{array}{c}8 \\
0 \\
i\end{array}$ & $\begin{array}{l}\stackrel{o}{+} \\
i\end{array}$ & $\stackrel{8}{-}$ & $\begin{array}{c}\stackrel{9}{+} \\
\mathrm{i}\end{array}$ & $\stackrel{n}{0}$ & 의 & ते & $\stackrel{\infty}{\sim}$ & $\stackrel{\text { \} }{\mathrm{i}}} &{\stackrel{\infty}{-}} &{\stackrel{\infty}{\circ}} &{\text { 울. }} &{\stackrel{\infty}{-}} &{\stackrel{\text { f }}{-}} &{\stackrel{\infty}{\infty}} &{\text { 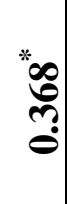 }} &{ } \\
{\hline \text { 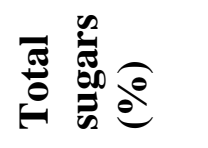 }} &{\vec{m}} &{\begin{array}{l}\infty \\
\stackrel{0}{0} \\
\end{array}} &{\begin{array}{c}0 \\
\stackrel{0}{\infty} \\
-\infty\end{array}} &{\begin{array}{l}\vec{\imath} \\
\stackrel{0}{0}\end{array}} &{\text { 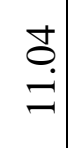 }} &{\text { ஃ. }} &{\text { 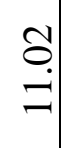 }} &{\underset{\dot{n}}{ \pm}} &{\begin{array}{l}\infty \\
n \\
n\end{array}} &{\text { 을 }} &{\text { 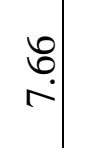 }} &{\stackrel{\overrightarrow{0}}{=}} &{\begin{array}{l}8 \\
\infty\end{array}} &{\text { n? }} &{\stackrel{?}{6}} &{\begin{array}{l}\stackrel{1}{\infty} \\
\infty\end{array}} &{\underset{\nabla}{\stackrel{\nabla}{b}}} &{\begin{array}{c}0 \\
i \\
i\end{array}} &{\stackrel{\substack{6 \\
0}}{0}} &{ } \\
{\hline \text { क्ष }} &{\text { 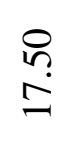 }} &{\text { 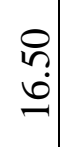 }} &{\begin{array}{l}0 \\
\stackrel{n}{2} \\
\grave{2}\end{array}} &{\begin{array}{l}0 \\
\stackrel{n}{2} \\
=\end{array}} &{\begin{array}{l}\stackrel{?}{+} \\
\dot{ \pm}\end{array}} &{\text { กิ }} &{\begin{array}{l}0 \\
\stackrel{n}{ \pm} \\
\pm\end{array}} &{\begin{array}{l}0 \\
\\
\infty\end{array}} &{\begin{array}{l}0 \\
\vdots \\
a\end{array}} &{\text { जी }} &{\stackrel{?}{=}} &{\begin{array}{l}\stackrel{0}{0} \\
\dot{ \pm} \\
-\end{array}} &{\text { ڤి }} &{\text { กิ? }} &{\begin{array}{l}\text { ?ִ } \\
\stackrel{0}{0}\end{array}} &{\begin{array}{l}\stackrel{0}{i} \\
\text { in }\end{array}} &{\begin{array}{l}\text { ?ִ } \\
\stackrel{0}{0}\end{array}} &{\begin{array}{l}0 \\
\infty \\
\infty \\
\infty\end{array}} &{\text { 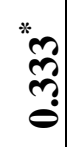 }} &{ } \\
{\hline \text { }} &{\text { 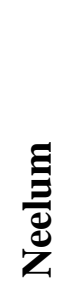 }} &{\text { 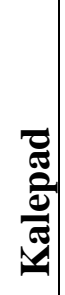 }} &{\text { 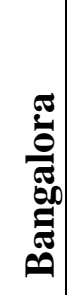 }} &{\text { 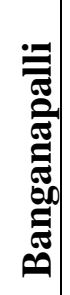 }} &{\text { 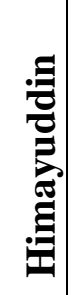 }} &{\begin{array}{l}\frac{\pi}{5} \\
\frac{\pi}{\mathbf{t}} \\
\mathbf{Z}\end{array}} &{\text { 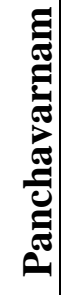 }} &{\text { 节 }} &{\text { 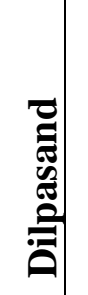 }} &{\text { 気 }} &{\text { : }} &{\text { 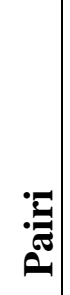 }} &{\text { : }} &{\text { 蔦 }} &{\text { 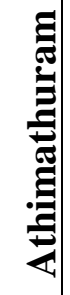 }} &{\text { 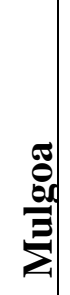 }} &{\text { 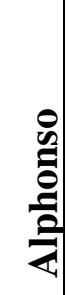 }} &{\text { 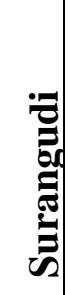 }} &{\sqrt{5}} &{6} \\
{\hline \dot{\infty} \not} &{-} &{N} &{n} &{\nabla} &{\text { (1) }} &{0} &{r} &{\infty} &{a} &{=} &{=} &{\simeq} &{\text { 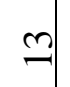 }} &{ \pm} &{\because} &{\text { 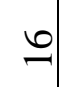 }} &{\text { 二 }} &{\stackrel{\infty}{-}} &{ } &{ } \\
$\hline
\end{tabular}


Table.3 Effect on off-season environmental changes in meteorological parameters

\begin{tabular}{|c|c|c|c|c|c|c|}
\hline 产 & $\tilde{\sigma}$ & $\stackrel{?}{r}$ & $\stackrel{b}{b}$ & $\stackrel{9}{r}$ & గु & $\vec{r}$ \\
\hline 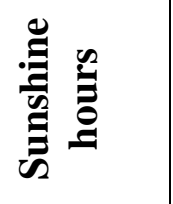 & $\begin{array}{l}n \\
0 \\
\infty \\
\infty\end{array}$ & 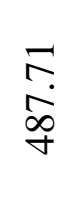 & $\begin{array}{l}\bar{n} \\
\text { oे } \\
\text { on }\end{array}$ & $\frac{\stackrel{Y}{f}}{\stackrel{g}{f}}$ & $\begin{array}{l}\vec{b} \\
\dot{0} \\
\dot{m}\end{array}$ & 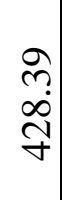 \\
\hline 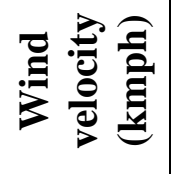 & $\hat{b}$ & $\begin{array}{l}\otimes \\
\dot{n}\end{array}$ & $\begin{array}{l}\stackrel{\infty}{1} \\
\stackrel{n}{n}\end{array}$ & $\begin{array}{l}\overrightarrow{0} \\
i n\end{array}$ & $\underset{\sim}{\sim}$ & $\begin{array}{l}\infty \\
0 \\
0 \\
0\end{array}$ \\
\hline 预 & $\stackrel{n}{+}$ & กे & $\begin{array}{l}0 \\
\dot{0}\end{array}$ & $\hat{\dot{8}}$ & $\underset{I}{ \pm}$ & $\begin{array}{l}\stackrel{\sim}{ } \\
\tilde{2}\end{array}$ \\
\hline 苞总 & $\stackrel{\infty}{\underset{\mathbb{}}{+}}$ & $\vec{n}$ & $\begin{array}{l}\infty \\
\stackrel{2}{\Omega}\end{array}$ & $\begin{array}{l}0 \\
\dot{\infty}\end{array}$ & $\stackrel{\stackrel{ }{\wedge}}{\circ}$ & $\stackrel{0}{i}$ \\
\hline 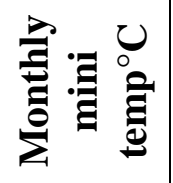 & तु & $\begin{array}{l}m \\
\ddot{n}\end{array}$ & $\begin{array}{l}\stackrel{0}{\sim} \\
\dot{+}\end{array}$ & $\begin{array}{l}0 \\
\text { in }\end{array}$ & $\vec{\otimes}$ & $\begin{array}{l}n \\
\stackrel{n}{2}\end{array}$ \\
\hline 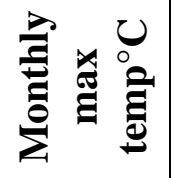 & $\frac{a}{m}$ & $\begin{array}{l}0 \\
\text { i }\end{array}$ & $\stackrel{\sim}{m}$ & $\hat{\text { ñ }}$ & बें & $\begin{array}{l}0 \\
\dot{m} \\
\dot{n}\end{array}$ \\
\hline 号 & 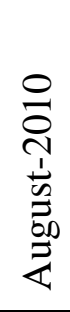 & 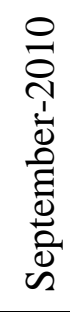 & 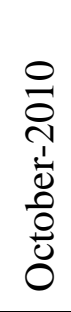 & $\begin{array}{l}0 \\
0 \\
\overline{0} \\
\frac{1}{\Delta} \\
\text { हี } \\
0 \\
0 \\
0\end{array}$ & 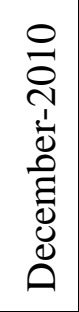 & 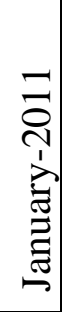 \\
\hline$\sum_{i}^{\circ}$ & - & $N$ & $n$ & $\nabla$ & $n$ & 0 \\
\hline
\end{tabular}

Sunshine hours ranged from (346.61 to 491.42). Although flower bud initiation in mango takes place during short days in the fall, off-season flowering during June is also observed near equator. Hence, it is difficult to call this species a short/long day plant. The 
climatic mystery is also twisted by the varietal differences as varieties like 'Neelum' flowers twice in Kanyakumari but once in north India. This effect is also found to be seen in this species as the trees which are grown in the eastern side of an orchard flowers a few days ahead of others. It clearly indicated that the trees found on the eastern side are found to receive longer hours of sunlight in fruits. Wind velocity ranged between (5.01 and 7.61 kmph). The State Horticultural Farm Kanyakumari which depicts that the diurnal variation in temperature is low with a range of $\left(6.2\right.$ to $\left.7.9^{\circ} \mathrm{C}\right)($ Table 3$)$ during the flowering months of June - August for off-season bearing. This is also supported by the prevalence of low night temperatures during that period when compared to other months. Ravishankar et al., (1979) reported that flower bud initiation in 'Alphonso' commenced in early October and reached a peak by mid November. The marked drop in the night temperature and relative humidity appeared to be favourable for fruit bud differentiation.

Many cultivars flower erratically in the low latitude tropics, providing continuously warm temperature with high soil and atmospheric moisture (Davenport, 2003). Researchers in Thailand had revealed that mango trees were in need of dry period for flower bud formation and flowering occured naturally after the dry and cool season (Rajan, 2009).

In conclusion, among the eighteen varieties Bangalora performance well in off-season mango production under Kanyakumari condition. While most of varieties which have shown preponderance of crop in the offseason can at best be termed only as mediocre from the stand point of quality, it is interesting to note that some trees of varieties in commercial cultivation such as Bangalora, Neelum, Kalepad, Banganapalli and Himayuddin etc. The possibility of stabilizing off-season cropping in commercial production of mango in certain tracts of south Tamil Nadu belt for off-season production. Whereas highly conducive environmental condition for off-season flowering and fruiting in Kanyakumari.

\section{References}

A.O.A.C. 1975. Official methods of analysis. Published by Association of Agricultural Chemists, Washington, D.C.

Anantanarayanan, K.K. and O.A.A. Pillai. 1968. Fruit maturity studies at Kanyakumari (Tamil Nadu), South. Indian Hort., 16(3\&4): 56-57.

Chacko, E.K. and Randhawa, G.S. 1971. Towards an understanding of the factors flowering in mango (Mangifera indica $\mathrm{L}$ ). Andhra Agri. J., 18: 226-236.

Damodaran, T., Medhi R.P., Gapil Dev, G., Damodaran, V., Rai, R.B. and Kavino, M. 2006. Identification of molecular markers linked with differential flowering behaviour of mangoes in Andaman and Nicobar Islands. Curr. Sci., 92(8): 10541056

Davenport, T.L. 2003. Management of flowering in three tropical and subtropical fruit tree species. Hort. Sci., 38: 1331-1335.

Gandhi, S.R. 1959. The Mango. Farm Bulletin, Indian Council of Agricultural Research., New Delhi.

Gangolly, S.R., S.L. Ranjit Singh Katyal and Daljit Singh. 1957. The Mango. Indian Council of Agricultural Research., New Delhi.

Hassan, A.M., M.A. Gihan and E. Sanaa, 2004. Maturation and heat units (G.D.D.) of some mango cultivars. Egypt. J. Appl. Sci., 19(5B): 553-571.

Kennedy, R.R., C. Swaminathan, S. Amutha, T. Rajesh and J. Prem Joshua. 2009a. Offseason flowering in mango - a biological wonder in Kanyakumari district, India. National level training cum seminar on off-season mango production. (Eds. Richard Kennedy et al.), Horticultural 
Research Station, Pechiparai, India, pp 44-47.

Kennedy, R., T. Thangaselvabai, J.A. Sheeba, J.P. Joshua and K.E.A. Aiyanathan. 2009b. Off-season production potential of mango in Kanyakumari. National seminar on production, post harvest technology and marketing of mango. (Eds. Balasubramanyan et al), Horticultural college and Research Institute, Periyakulam, India, pp 105-108.

Khattab, M.M., G.M. Haseeb, A.E. Shaban and M.A. Arafa. 2006. Effect of paclobutrazol and potassium nitrate on flowering and fruiting of Ewais and Sediek mango trees. Bull. Fac. Agric., Cairo Univ., 57(1): 107-124.

Lakshminarayanareddy, M., G. Satyanarayana reddy, T. Sureshkumar and A. Bhagwan. 2009. Studies on the performance of offseason cropping mango varieties in Telungana regions of Andhra Pradesh. National seminar on production, post harvest technology and marketing of mango. (Eds. Balasubramaniyam et al), Horticultural college and Research Institute, Periyakulam, India pp 34-36.

Lysander, M. and K. Pillai, 1957. On off-season mango production. South. Indian Hort., 5: 20-24.

Naik, K.C. 1949. South Indian fruits and their culture. P. Varadachary and Co., Madras.

Parthiban, S., K. Balakrishnan, C. Rajamanickam and M. Selvarajan. 2009. Flowering behavior in mango. National seminar on production, post harvest technology and marketing of mango. (Eds. Balasubramaniyam et al), Horticultural college and Research Institute, Periyakulam, India, pp 105-
108.

Rajan, S. 2009. Off-season mango production: present status and future scope. National level training cum seminar on off-season mango production. (Eds. Richard Kennedy et al.), Horticultural Research Station, Pechiparai, India pp 1-4.

Ravishankar, H., M.M. Rao and K.M. Bojappa. 1979. Fruit bud differentiation in mango 'Alphonso' and 'Totapuri' under mid tropical rainy conditions. Scientia. hort., 10: $95-99$.

Shaban, A.E.A. 2009. Vegetative growth cycles of some mango cultivars in relation to flowering and fruiting. World J. Agri. Sci., 5(6): 751-759.

Singh, L.B. 1957. Biennial bearing in mango. Hort Adv., 1: 7-22.

Singh, R.N. Mango. 1996. New Delhi, ICAR.

Somogyi, N. 1952. Notes on sugar determination. J. Biol. Chem., 200: 145154.

Srinivasan, C. and K.G. Shanmugavelu. 1971. Proximate composition of certain off- season mango varieties of Tamil Nadu. South Indian Hort., 19: 24-28.

Sundaram. R. 2007. King of fruits' becoming popular in Erode. The Hindu, 27.11.07.

Sundararaj, J.S., S. Muthuswamy and N. Raghavan. 1972. Off-season cropping of mango. Symposium on mango and mango culture. Acta Hort., 24: 189-191.

Velappan, D. and C.A.S. Shankar. 2001. Agriculture citizens report on the state of development and environment in Kanyakumari District. R. R. Daniel, A. D. Sobhnaraj, M. Jazer Jebanesan and D. Thomas Franko (Ed.) South vision pp 1431.

\section{How to cite this article:}

Kaviarasu, A., S. Balakrishnan and Indirakumar, K. 2017. Environmental Influence under Offseason Production on Yield and Quality Attributes of Mango. Int.J.Curr.Microbiol.App.Sci. 6(2): 494-502. doi: http://dx.doi.org/10.20546/ijcmas.2017.602.056 